\title{
Traditional, forgotten and new left ventricular systolic function parameters on a 64-row multidetector cardiac computed tomography: A reproducibility study
}

\author{
Maciej Sosnowski ${ }^{1}$, Rafał Młynarski ${ }^{2}$, Michał Tendera ${ }^{3}$ \\ ${ }^{1}$ Unit for Noninvasive Cardiovascular Diagnostics, $3^{\text {rd }}$ Chair of Cardiology, \\ Medical University of Silesia, Katowice, Poland \\ ${ }^{2}$ Unit for Noninvasive Cardiovascular Diagnostics, Department of Electrocardiology, \\ Upper Silesian Heart Centre, Katowice, Poland \\ ${ }^{3} 3^{\text {rd }}$ Chair and Department of Cardiology, Medical University of Silesia, Katowice, Poland
}

\begin{abstract}
Background: Multidetector computed tomography angiography (MDCT) can provide data regarding cardiac function if a retrospective scanning is applied. We aimed at examination of the reproducibility of traditional and more sensitive parameters of the left ventricular (LV) contractility by means of a 64-row CT in order to establish errors of measurement and to determine limits that allow for a reliable detection of their changes.

Methods and Results: A random sample of 25 individuals, including 15 females (aged $64 \pm 13$ years) and 10 males (54 \pm 13 years), who had MDCT examination were retrospectively included in this study. Data reconstructions were performed on a dedicated workstation. In each case, axial image series were created with a 10\% step from 0\% to 90\% of the RR interval using a $2 \mathrm{~mm}$ slice thickness. LV volume was determined in each phase. Detailed LV volume changes within phases were analyzed to determine the largest difference between the neighbor phases (peak ejection volu$m e, P E V, m L$ ) during systole and to calculate the peak ejection rate (PER i.e. PEV/phase duration $\left[1 / 10^{\text {th }}\right.$ of $R R$ interval], $\left.m L / s\right)$. The derived parameters were calculated as the PER normalized for LVEDV $(P E R-V, 1 / s)$, the PER normalized for $L V M(P E R-M, m L / g \times s)$ and the PER normalized for LVEDV times the PER normalized for LVM product $\left(P E R-V M, \mathrm{ml} / \mathrm{g} \times \mathrm{s}^{2}\right)$. Considering the errors percentages, the respective values for intra- and inter-observer errors were around $5 \%$ and $8 \%$ for standard LV systolic measures. The percentage intra-observer errors' ranged between $-7.8 \%$ and $-10.8 \%$, and the inter-observer errors' ranged between $-11.8 \%$ and $-15.7 \%$ for both PEV and PER. For the same reader, the percentage errors ranged between $-8.7 \%$ and $+11.9 \%$ for $P E R-V,-10 \%$ and $+12.7 \%$ for PER-M and $-18.2 \%$ and $+24 \%$ for PER-VM. For the independent reader the corresponding values were $-15.2 \%$ and $+15.5 \%,-12.3 \%$ and $+16.3 \%$, and $-26.6 \%$ and $+30.9 \%$. The intra-class coefficients for repeated measurements for both the same reader (intra-observer) or independent reader (inter-observer) did reach values above 0.9 and around 0.8 , respectively.
\end{abstract}

Conclusions: We concluded that traditional LV systolic parameters, as well as more sensitive measures of cardiac contractility could be determined reliably by means of a 64-row MDCT. The errors for global LV systolic function measures amount to about 5\%, for PEV and PER about $15 \%$ and for the PER-derived parameters about 25\%. The measurement errors established might help to assess the significance of changes in repeated MDCT examinations. (Cardiol J 2013; 20, 4: 385-393)

Key words: left ventricular systolic function, peak ejection rate, cardiac computed tomography

Address for correspondence: Maciej Sosnowski, MD, PhD, Unit for Noninvasive Cardiovascular Diagnostics,

$3^{\text {rd }}$ Chair of Cardiology, Medical University of Silesia, ul. Ziołowa 47, 40-635 Katowice, Poland, tel: +48 323598663 , fax: +4832356986 63, e-mail: maciej.sosnowski@gmail.com 


\section{Introduction}

Coronary computed tomography (CT) angiography is an established noninvasive method for coronary arteries imaging in patients with symptoms suggestive of coronary artery disease, both for diagnostic and prognostic purposes [1,2]. In a case of the retrospective scanning, a multi-detector CT (MDCT) examination permits the measurement of volumes, and assessment of cardiac function [3]. Apart from diagnostic information, the left ventricular (LV) function evaluation adds incremental prognostic value over coronary artery evaluation [4]. It has already been documented that cardiac MDCT allows for a reliable and reproducible assessment of LV volume, hence the most common $\mathrm{LV}$ global systolic function measure, i.e. LV ejection fraction (LVEF) [5]. However, LVEF has certain limitations for LV contractility determination [6, 7]. We aimed at examination of the reproducibility of more detailed parameters of LV systolic function by means of a MDCT in order to establish errors of their measurements and to determine error-related limits for reliable detection of their changes.

\section{Methods}

\section{Study patients}

A random sample of 25 individuals, including 15 female patients (mean age $64 \pm 13$ years) and 10 male patients (mean age $54 \pm 13$ years), who had cardiac MDCT examination performed between June 2007 and March 2012, were retrospectively included in this study. The reasons for the cardiac MDCT examination were either the evaluation of coronary arteries, coronary stents or aorto-coronary anastomoses patency, or detection of ascending aorta dissection. In a few, cardiac MDCT examination was a part of investigation procedures before aortic valve implantation, either surgical or transcutaneous. Exclusion criteria were: cardiac arrhythmias, including atrial fibrillation or frequent premature beats, known allergy to iodine-contrast agents, renal impairment (serum creatinine $>150 \mathrm{mmol} / \mathrm{L}$ ).

All patients gave their informed consent for the MDCT examination (for clinical indications). The study conformed to the revised Helsinki Declaration upon ethical principles for medical research involving human subjects, amended in 2008 [8]. Local ethics committee approval was not required taking into account the retrospective and anonymous design of the study.

\section{Cardiac MDCT study}

Cardiac MDCT examinations were performed with a 64 scanner (Aquilion, Toshiba, Japan). The detector collimation was $64 \times 0.5 \mathrm{~mm}$ with the rotation time of $0.4 \mathrm{~s}$. The CT pitch factor was between 0.2 and 1.0. The tube voltage and current were set taking into account the patient's body mass index (BMI): $120 \mathrm{kV}$ and $330 \mathrm{~mA}$ for BMI $<24 \mathrm{~kg} / \mathrm{m}^{2}, 135 \mathrm{mV}$ and $380 \mathrm{~mA}$ for BMI between 24 and 30 , and $135 \mathrm{kV}$ and $430 \mathrm{~mA}$ in those with BMI $>30$. Depending on the scanning time, a total volume of $90 \mathrm{~mL}$ to $130 \mathrm{~mL}$ of non-ionic contrast (iomeprol, Iomeron 400, Bracco, UK or iopromide, Ultravist 370, Schering, Germany) was injected into the antecubital vein at a flow rate of $4-5 \mathrm{~mL} / \mathrm{s}$.

The retrospective ECG-gated scanning of the heart, preceded by the Sure-Start monitoring at the start scan position, was performed. The scanning was triggered once the descending aorta opacity reached 180 Hounsfield units. Cardiac images ranging usually from the ascending aorta to the apex were acquired during a single breath-hold of approximately $10 \mathrm{~s}(\mathrm{n}=20)$. In some cases the window was extended towards aortic arc (post-coronary artery bypass grafting, $\mathrm{n}=3$, aortic stenosis, $\mathrm{n}=2$ ). Depending on the scanning span, the effective dose ranged between 12-18 milliSieverts (mSv) for usual cardiac MDCT (coronary angiography), and between $15 \mathrm{mSv}$ and $33 \mathrm{mSv}$ for cardiac and thoracic aorta scanning.

Reconstructions of data were performed on dedicated workstation (Vitrea2, Vital Images, USA, version 5.1). In each case, ten axial image series were created with a $10 \%$ step from $0 \%$ to $90 \%$ of the RR interval, using a $2 \mathrm{~mm}$ slice thickness in order to reduce the amount of data.

\section{Data analysis}

$\mathrm{LV}$ volume was determined in each phase semi-automatically using the commercially available software under visual inspection and manual correction, if necessary. Phases with the largest and the smallest LV volume defined the LV end-diastole and LV end-systole with their corresponding volumes (LVEDV and LVESV, $\mathrm{mL}$ ). LVEF (\%) was calculated by using commonly used formula. LV mass (LVM, g) was calculated as the difference between epicardial and endocardial volumes multiplied by a factor of $1.04[\mathrm{~g} / \mathrm{mL}]$. Detailed LV volume changes within phases of cardiac systole were analyzed to determine the largest difference 


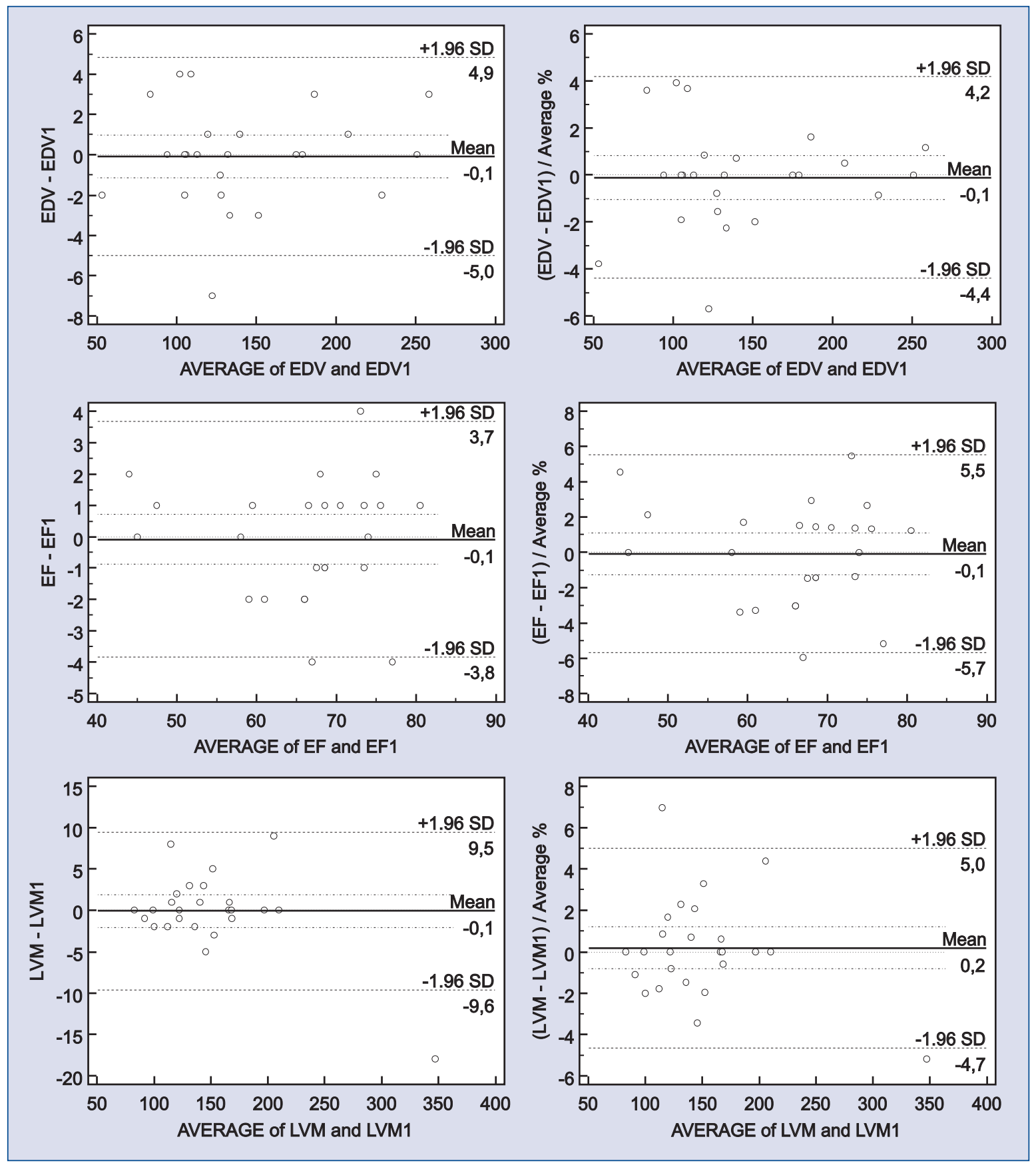

Figure 1. Bland-Altman plot of reproducibility of global systolic function parameters (intra-observer).

between the neighboring phases (peak ejection volume, $\mathrm{PEV}, \mathrm{mL}$ ) during systolic phases and to calculate the peak ejection rate (PER i.e. PEV/ /phase duration [1/10 ${ }^{\text {th }}$ of $\mathrm{RR}$ interval], $\mathrm{mL} / \mathrm{s}$ ) [9]. The derived parameters were calculated as the PER normalized for LVEDV (PER-V, 1/s), the PER normalized for LVM (PER-M, $\mathrm{mL} / \mathrm{g} \times \mathrm{s}$ ) and the PER normalized for LVEDV times the PER normalized for LVM product (PER-VM, $\left.\mathrm{mL} / \mathrm{g} \times \mathrm{s}^{2}\right)$.
All data were measured twice by the same reader (MS) or by another reader blinded to the other measurements (RM) at least 6 weeks apart from the first evaluation.

\section{Statistical analysis}

Measurement errors were quantified by means of Bland-Altman method [10]. For each parameter the intra-class coefficients were calculated. 


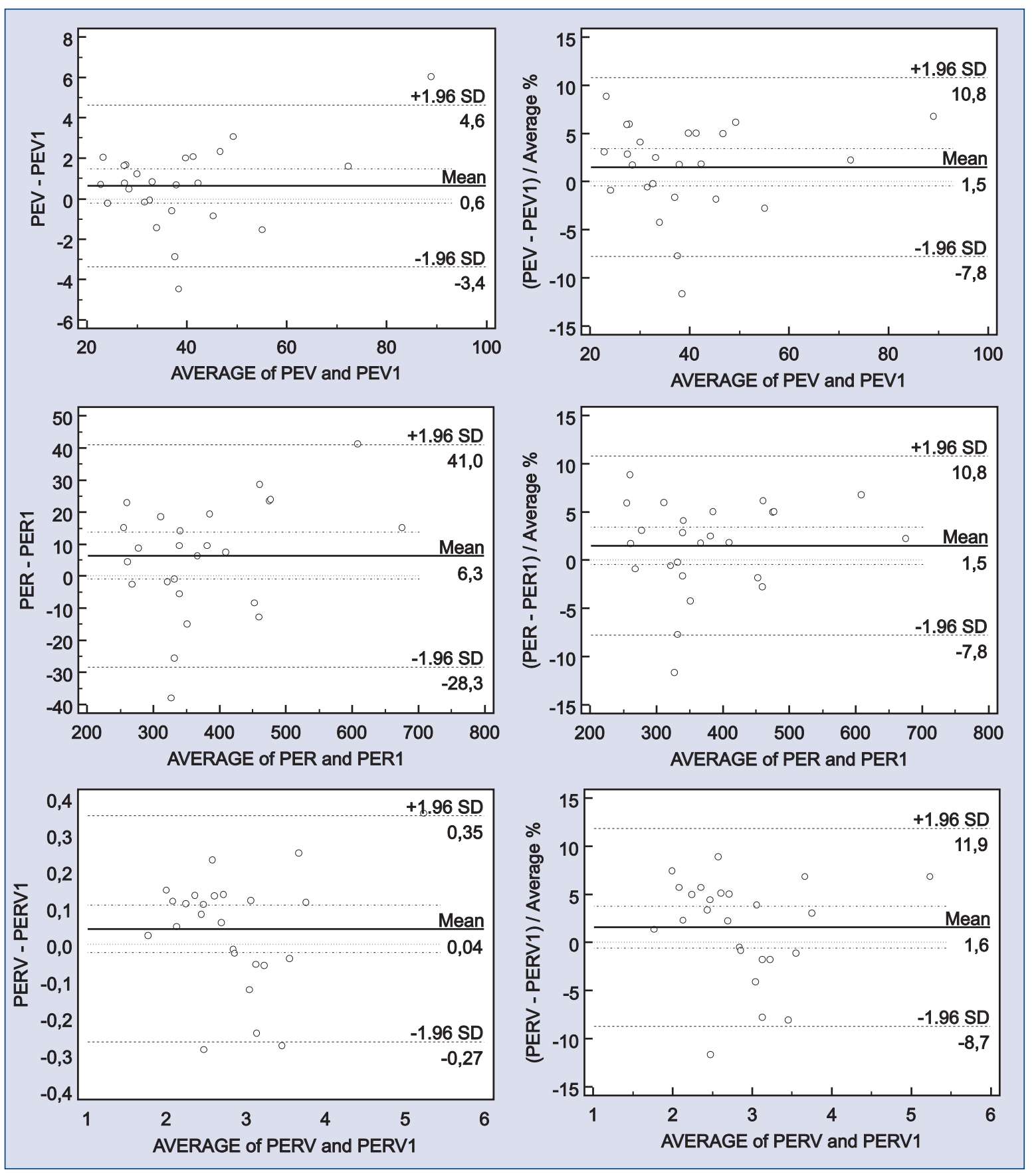

Figure 2. Bland-Altman plot of reproducibility of peak ejection volume-derived parameters (intra-observer).

\section{Results}

The detailed results of measurements are displayed in Figures 1-4. For repeated measurements by the same reader, the mean difference of the global LV systolic function parameters $( \pm 1.96 \mathrm{SD})$ was less than $5 \mathrm{~mL}$ for EDV, $3.8 \%$ for EF and $9.6 \mathrm{~g}$ for LVM (Fig. 1). The inter-reader errors reached $7 \mathrm{~mL}, 5.0 \%$ and $19.1 \mathrm{~g}$, respectively (Fig. 3). While considering the errors percentages, the respec- tive values for intra- and inter-observer errors were $4.3 \%, 5.6 \%$ and $5.2 \%$, and $5.3 \%, 10.6 \%$ and $8.8 \%$.

For the PEV and PER, the mean absolute difference between the two measurements reached (intra-observer) $4 \mathrm{~mL}$ and $34.7 \mathrm{~mL} / \mathrm{s}$, and (inter-observer) $4.8 \mathrm{~mL}$ and $52.6 \mathrm{~mL} / \mathrm{s}$ (Figs. 2, 4). The corresponding percentage of intra-observer errors' range were quantified as lying between $-7.8 \%$ and $-10.8 \%$, and the inter-observer errors' range between $-11.8 \%$ and $-15.7 \%$ for both PEV and PER. 

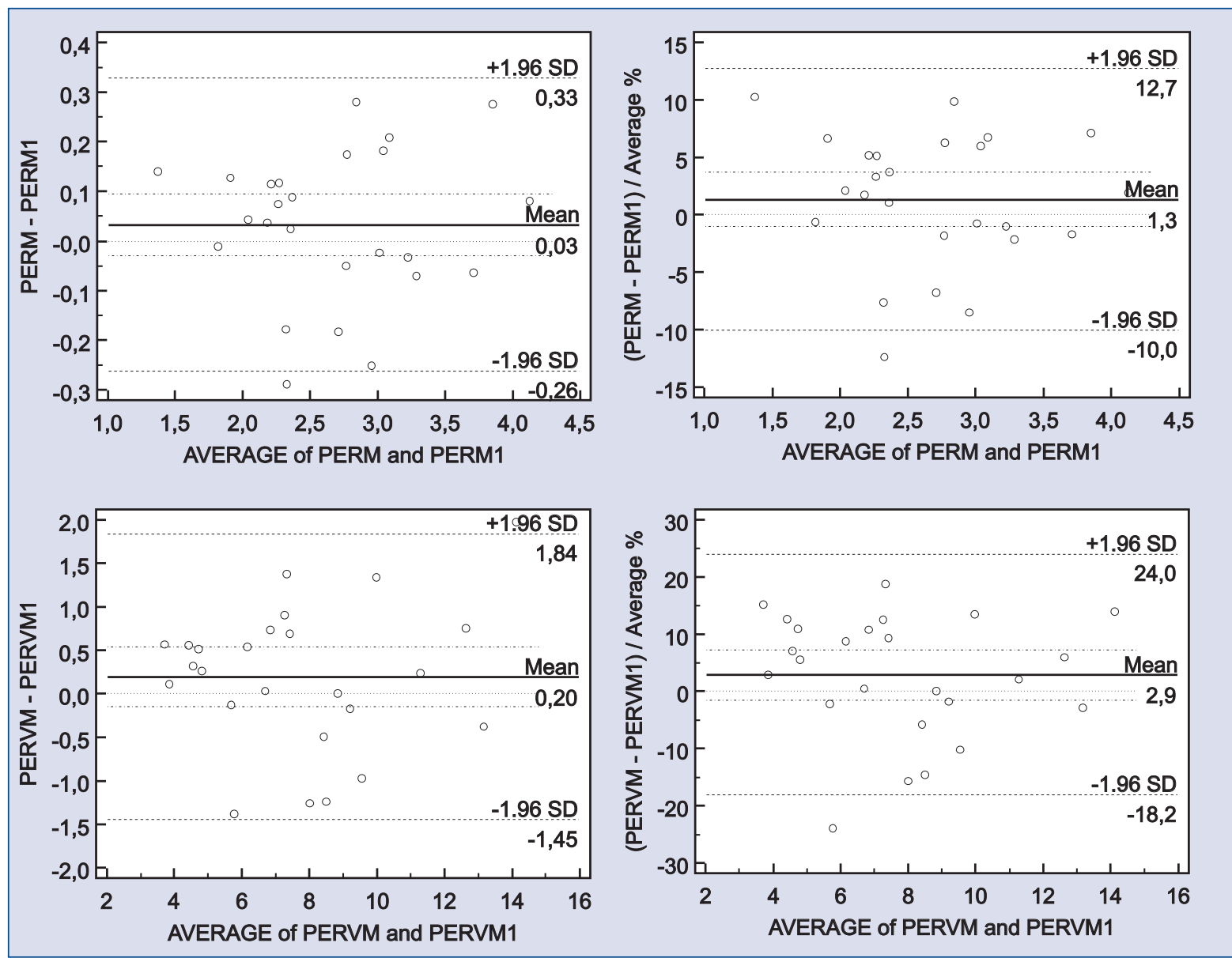

Figure 2 (continued). Bland-Altman plot of reproducibility of peak ejection volume-derived parameters (intra-observer).

The absolute PER-derived parameter errors were quantified as reaching $0.31 \mathrm{1} / \mathrm{s}$ for PER-V, $0.30 \mathrm{~mL} / \mathrm{g} \times \mathrm{s}$ for PER-M, and $1.45 \mathrm{~mL} / \mathrm{g} \times \mathrm{s}^{2}$ for PER-VM for the same reader (Fig. 2), and $0.361 / \mathrm{s}$ for PER-V, $0.30 \mathrm{~mL} / \mathrm{g} \times \mathrm{s}$ for PER-M, and $1.62 \mathrm{~mL} / \mathrm{g} \times \mathrm{s}^{2}$ for PER-VM for the independent second reader (Fig. 4). For the same reader, the corresponding percentage errors ranged between $-8.7 \%$ and $+11.9 \%$ for PER-V, $-10 \%$ and $+12.7 \%$ for PER-M and $-18.2 \%$ and $+24 \%$ for PER-V $\times$ PER-M (Fig. 2 ). For the independent reader (inter-observer errors) the corresponding values were $-15.2 \%$ and $+15.5 \%$, $-12.3 \%$ and $+16.3 \%$, and $-26.6 \%$ and $+30.9 \%$ (Fig. 4 ). The intraclass correlation coefficients for repeated measurements for both the same reader and independent reader are presented in Table 1.

\section{Discussion}

Cardiac function is a basic clinical variable that guides diagnosis, therapeutic decisions, as well as prognosis. In practice, it is assessed by means of echocardiography, however, in certain cases, inadequate acoustic window and an operator-dependent error, as well as not optimal reproducibility might limit its reliability [11-13]. On the other hand, the use of the so-called "gold" standard method, i.e. nuclear magnetic resonance, is limited by a relatively low availability and costs. Two other methods, i.e. gated single photon emission $\mathrm{CT}$ and cardiac MDCT, lie in between. Cardiac MDCT is an acceptable alternative method for cardiac function evaluation in patients who, for various reasons, have contra-indications for "gold standard" method or who present with features that limit a reliable cardiac function assessment. As long as the retrospective scanning is used (mostly for coronary arteries, aortic valve and ascending aorta evaluation), the data stored allows for determination of cardiac chambers' volumes and their changes over cardiac cycle. As the X-rays exposure cannot be ignored in a retrospective scanning [14], reports about all possible features, including cardiac function, do seem mandatory. 


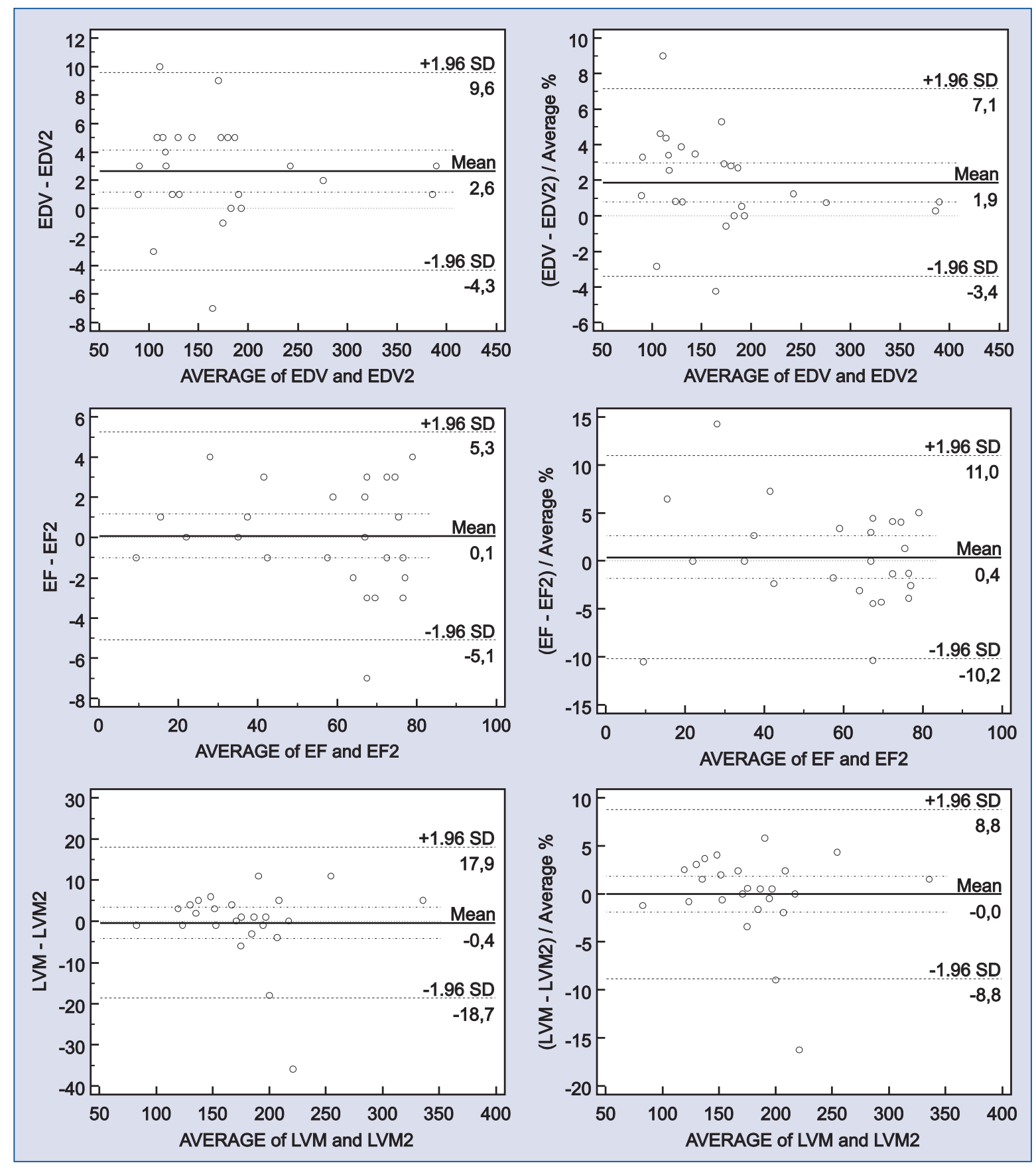

Figure 3. Bland-Altman plot of reproducibility of global systolic function parameters (inter-observer).

Determination of the errors of measurement of any parameter does seem obligatory if the method is going to be used for diagnostic purposes. Such errors can partly explain the differences between MDCT and other methods, including "golden" or "reference" methods, usually echocardiography. We did not address this issue, as magnetic resonance imaging was not available at our center.

Results of our study indicate that more sensitive cardiac contractility measures could be determined using a 10-phase retrospective reconstructions. However, as the error of measurements might be considered high (25\%), a clear change of systolic function might not out-weight a more reproducible LVEF assessment. We are aware that more frequent sampling (20 phases, each of $5 \%$ of R-R interval) might provide a more precise measurement [15, 16], albeit at the cost of the substantial increase of the time needed for reconstructions and analysis. 

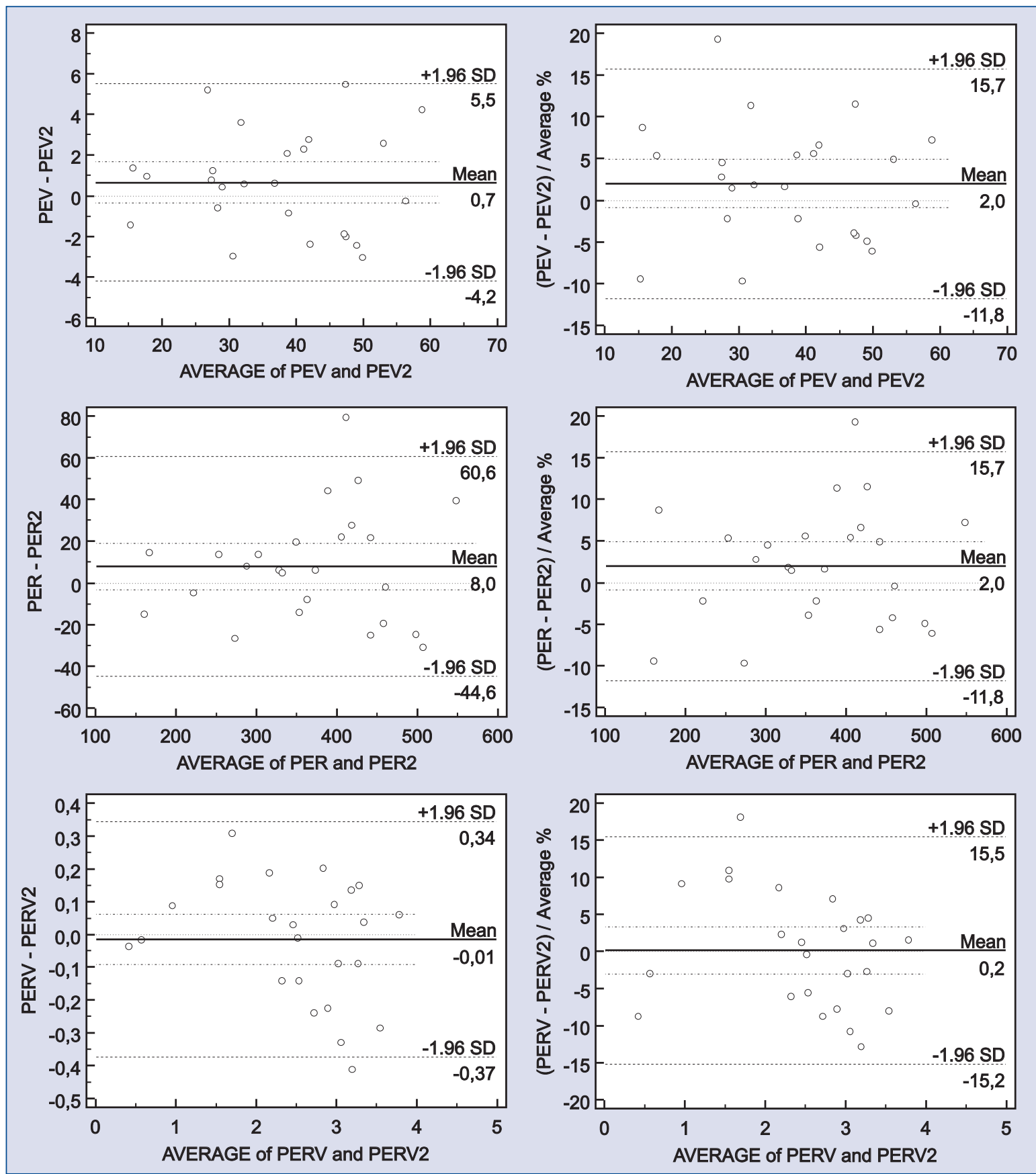

Figure 4. Bland-Altman plot of reproducibility of peak ejection volume-derived parameters (inter-observer).

We were unable to assess the qualitative comparisons since normal limits for several parameters had not been established as yet. Also, there is no data from previous studies that might serve as a source of normal limits. As cardiac CT examination cannot be performed in apparently healthy individuals for ethical reasons, data regarding lower and upper normal limits cannot be actually established.
We would like to emphasize that in the present study we introduced a new parameter of systolic function, i.e. PEF normalized for LVEDV times the PEF normalized for LVM. In contrast to commonly used parameters, such a new measurement combines effects of both pressure- and volume-related loads. In addition, PEF normalized for LVEDV and LVM does not need to be indexed for body surface area or height. However, changes amounting to 

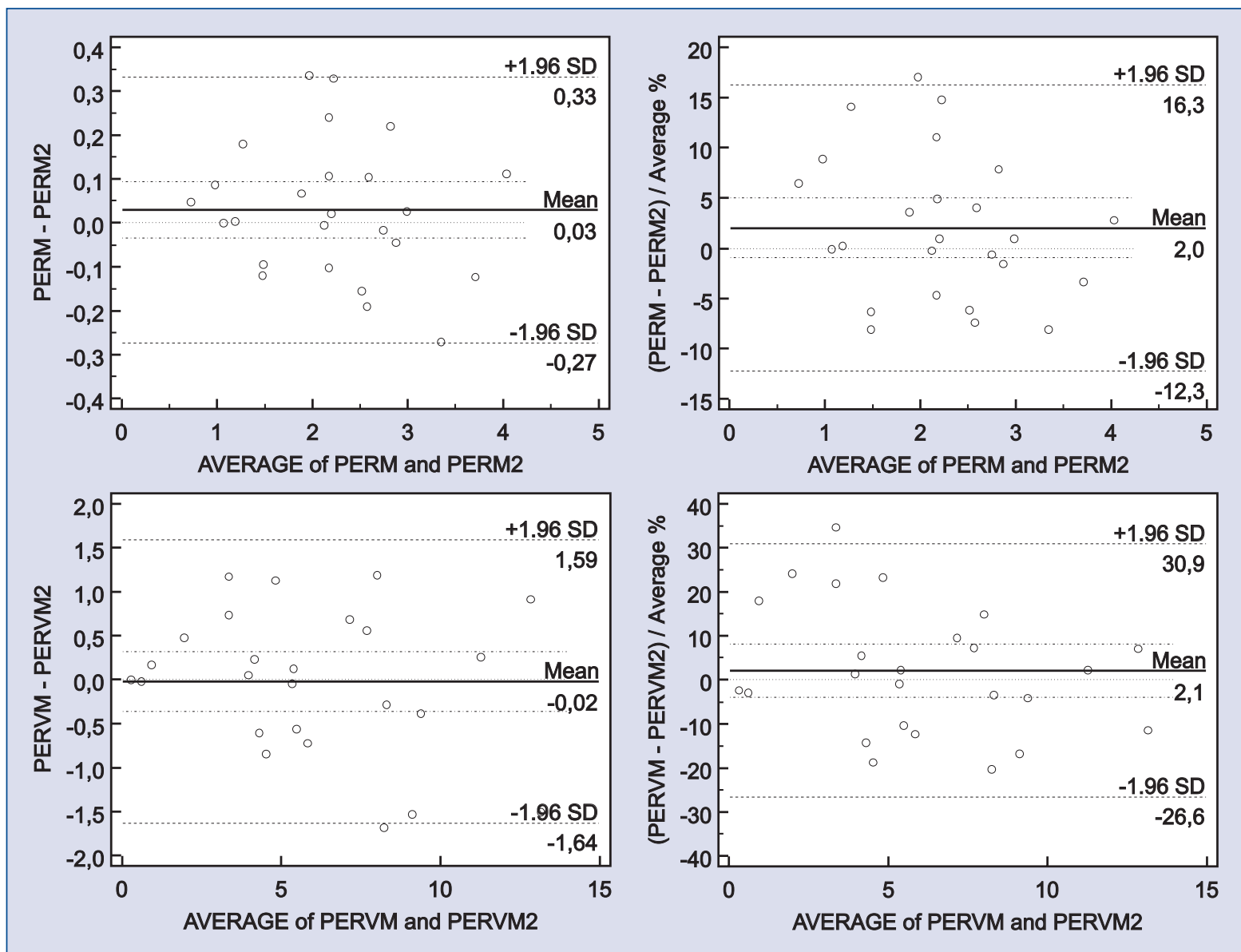

Figure 4 (continued). Bland-Altman plot of reproducibility of peak ejection volume-derived parameters (inter-observer).

Table 1. Reproducibility of left ventricular systolic function parameters as estimated by means of intra-class coefficients.

\begin{tabular}{lcc}
\hline Parameter & $\begin{array}{c}\text { Intra-observer } \\
\text { ICC [95\% confidence interval] }\end{array}$ & $\begin{array}{c}\text { Inter-observer } \\
\text { ICC [95\% confidence interval] }\end{array}$ \\
\hline EDV & $0.99[0.997-0.999]$ & $0.99[0.997-0.999]$ \\
LVEF & $0.98[0.956-0.991]$ & $0.99[0.983-0.997]$ \\
LVM & $0.99[0.991-0.998]$ & $0.98[0.964-0.993]$ \\
PEV & $0.99[0.979-0.996]$ & $0.98[0.954-0.991]$ \\
PER & $0.98[0.968-0.994]$ & $0.96[0.921-0.984]$ \\
PER-V & $0.99[0.980-0.996]$ & $0.98[0.957-0.991]$ \\
PER-M & $0.97[0.943-0.989]$ & $0.98[0.963-0.993]$ \\
PER-V $\times$ PER-M & $0.96[0.910-0.982]$ & $0.97[0.943-0.989]$ \\
\hline
\end{tabular}

The intra-class correlation coefficient (ICC) value $>0.9$ indicates almost perfect reproducibility; see abbreviations in the text

over $25 \%$ of basal calculation are necessary to be considered significant.

\section{Conclusions}

Traditional LV systolic parameters, as well as more detailed measures can be reliably determined by means of a 64-row MDCT. The errors for global LV sy- stolic function measures were found in about $5 \%$, for PEV and PER in about $15 \%$ and for the PER-derived parameters in about $25 \%$. The measurement errors established might help to assess the significance of changes in repeated MDCT examinations.

Conflict of interest: none declared 


\section{References}

1. Gao D, Ning N, Guo Y, Ning W, Niu X, Yang J. Computed tomography for detecting coronary artery plaques: a meta-analysis. Atherosclerosis, 2011; 219: 603-609.

2. Woods KM, Fischer C, Cheezum MK, Hulten EA, Nguyen B, Villines TC. The prognostic significance of coronary CT angiography. Curr Cardiol Rep, 2012; 14: 7-16.

3. Lin FY, Min JK. Assessment of cardiac volumes by multidetector computed tomography. J Cardiovasc Comput Tomogr, 2008; 2: 256-262.

4. Chow BJ, Wells GA, Chen L et al. Prognostic value of 64-slice cardiac computed tomography severity of coronary artery disease, coronary atherosclerosis, and left ventricular ejection fraction. J Am Coll Cardiol, 2010; 55: 1017-1028.

5. San Román JA, Candell-Riera J, Arnold R et al. Quantitative analysis of left ventricular function as a tool in clinical research. Theoretical basis and methodology. Rev Esp Cardiol, 2009; 62: 535-551.

6. Mahler F, Ross J Jr, O'Rourke RA, Covell JW. Effects of changes in preload, afterload and inotropic state on ejection and isovolumic phase measures of contractility in the conscious dog. Am J Cardiol, 1975; 35: 626-634.

7. Slutsky R, Watkins J, Peterson K, Karliner J. The response of left ventricular function and size to atrial pacing, volume loading and afterload stress in patients with coronary artery disease. Circulation, 1981; 63: 864-870.

8. Declaration of Helsinki. WMA. From: http://www.wma.net/e/ ethicsunit/helsinki.htm.
9. Hammermeister KE, Brooks RC, Warbasse JR. The rate of change of left ventricular volume in man. I. Validation and peak systolic ejection rate in health and disease. Circulation, 1974; 49: 729-738.

10. Bland JM, Altman DG. Statistical methods for assessing agreement between two methods of clinical measurement. Lancet, 1986; 1: 307-310.

11. Bernard Y, Meneveau N, Boucher S et al. Lack of agreement between left ventricular volumes and ejection fraction determined by two-dimensional echocardiography and contrast cineangiography in postinfarction patients. Echocardiography, 2001; 18: 113-122.

12. Picard MH, Popp RL, Weyman AE. Assessment of left ventricular function by echocardiography: a technique in evolution. J Am Soc Echocardiogr, 2008; 21: 14-21.

13. Thorstensen A, Dalen H, Amundsen BH, Aase SA, Stoylen A. Reproducibility in echocardiographic assessment of the left ventricular global and regional function, the HUNT study. Eur J Echocardiogr, 2010; 11: 149-156.

14. Halliburton SS, Abbara S, Chen MY et al.; Society of Cardiovascular Computed Tomography. SCCT guidelines on radiation dose and dose-optimization strategies in cardiovascular CT. J Cardiovasc Comput Tomogr, 2011; 5: 198-224.

15. Henneman MM, Schuijf JD, Jukema JW et al. Comprehensive cardiac assessment with multislice computed tomography: Evaluation of left ventricular function and perfusion in addition to coronary anatomy in patients with previous myocardial infarction. Heart, 2006; 92: 1779-1783.

16. Stolzmann P, Scheffel H, Leschka S et al. Reference values for quantitative left ventricular and left atrial measurements in cardiac computed tomography. Eur Radiol, 2008; 18: 1625-1634. 